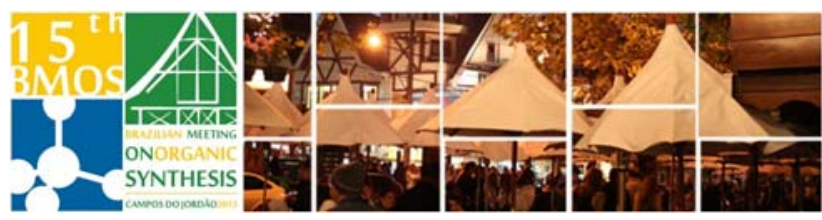

\title{
Borylation, Silylation, Fluorination and Fluoroalkylation
}

\author{
John F. Hartwig \\ University of California, Dept. of Chemistry, 718 Latimer Hall, Berkeley, CA 94720-1460, USA \\ ${ }^{\star} E$-mail: ihartwig@berkeley.edu
}

Keywords: C-H bond functionalization, Cross Coupling, Catalysis

\section{Abstract Speech}

This lecture will present a combination of methodology and underlying principles addressing the functionalization of $\mathrm{C}-\mathrm{H}$ bonds of arenes and alkanes with main group reagents and the fluorination and fluoroalkylation of arenes, heteroarenes, and organic halides. The development of reactions that regioselectively convert aromatic and aliphatic $\mathrm{C}-\mathrm{H}$ bonds to aryl and alkyl borane and silane derivatives will be presented, along with reactions of these main group species to form a series of functionalized products. The development of reactions of aryl and heteroaryl halides that form fluoroarenes and fluoroalkylarenes also will be presented. The relationships between $\mathrm{C}$ $\mathrm{B}, \mathrm{C}-\mathrm{Si}, \mathrm{C}-\mathrm{F}$ and $\mathrm{C}-\mathrm{R}_{\mathrm{f}}$ bond-forming processes will be discussed.

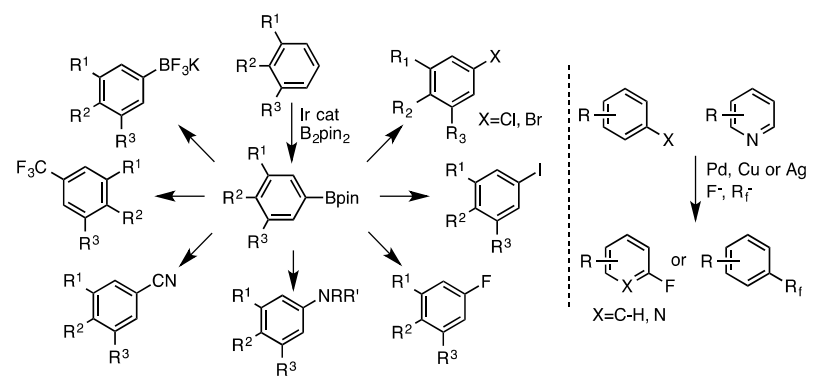

\section{REFERENCES}

${ }^{1}$ Mkhalid, I. A. I.; Barnard, J. H.; Marder, T. B.; Murphy, J. M.; Hartwig, J.

F. Chem. Rev. 2010, 110, 890.

${ }^{2}$ Simmons, E. M.; Hartwig, J. F. Nature 2012, 483, 70

${ }^{3}$ Morimoto, H.; Tsubogo, T.; Litvinas, N. D.; Hartwig, J. F. Angew. Chem.

Int. Ed. 2011, 50, 3793. 